\title{
Period-Doubling/Symmetry-Breaking Mode Interactions in Iterated Maps
}

\author{
P.J. Aston* and H. Mir \\ Department of Mathematics \\ University of Surrey \\ Guildford \\ Surrey GU2 7XH \\ UK
}

December 6, 2011

\begin{abstract}
We consider iterated maps with a reflectional symmetry. Possible bifurcations in such systems include period-doubling bifurcations (within the symmetric subspace) and symmetry-breaking bifurcations. By using a second parameter, these bifurcations can be made to coincide at a mode interaction. By reformulating the period-doubling bifurcation as a symmetry-breaking bifurcation, two bifurcation equations with $Z_{2} \times Z_{2}$ symmetry are derived. A local analysis of solutions is then considered, including the derivation of conditions for a tertiary Hopf bifurcation. Applications to symmetrically coupled maps and to two coupled, vertically forced pendulums are described.
\end{abstract}

PACS codes: $02.30 \mathrm{Oz}, 05.45 \mathrm{Ra}$

Keywords: Mode interaction, period-doubling bifurcation, symmetry-breaking bifurcation, coupled maps

\section{Introduction}

We consider iterated maps of dimension two or more which have a reflectional symmetry.

Two types of bifurcations which can occur in such maps from a symmetric fixed point are

(i) a period-doubling bifurcation (of symmetric solutions) and (ii) a symmetry-breaking bifurcation of fixed points. In a two-parameter system, these two types of bifurcation can be made to coincide, giving rise to a mode interaction. It is this type of mode interaction that we study. We note that a Hopf bifurcation in maps without symmetry gives rise

${ }^{*}$ Corresponding author: Dr Philip J Aston, Department of Mathematics, University of Surrey, Guildford, Surrey GU2 7XH, UK. Tel: +44 1483 682631. Fax: +44 1483 686071. Email: P.Aston@surrey.ac.uk 
to normal form equations where the Hopf bifurcation occurs as a $Z_{2}$ symmetry-breaking bifurcation of fixed points in the amplitude equation. Thus, a mode interaction between a period-doubling bifurcation and a Hopf bifurcation could also be studied using the approach described here.

Mode interactions in vector fields with various symmetry groups have been studied including $Z_{2} \times Z_{2}[1,2], O(2)[3,4]$ and $O(3)$ [5]. Mode interactions in iterated maps have also been considered in various forms. A codimension 2 point with critical eigenvalues +1 and -1 (in maps without symmetry) is known as the fold-flip bifurcation and has been considered by Kuznetsov, Meijer and van Veen [6]. Kuznetsov and Meijer [7] considered a range of possible codimension 2 situations associated with bifurcation of fixed points of maps. In particular, they considered 11 cases associated with two critical eigenvalues, but in systems without symmetry, deriving normal form equations in each case. In a subsequent paper, they also consider further codimension 2 cases with at least one pair of complex critical eigenvalues [8]. Since a Hopf bifurcation gives rise to a normal form for the amplitude which has $Z_{2}$ symmetry, the case that they consider of a complex pair of critical eigenvalues together with an eigenvalue of -1 is close to the situation that we consider. However, their normal form immediately has $Z_{2} \times Z_{2}$ symmetry due to the normal form symmetry associated with period-doubling bifurcations, and so they do not consider equations in the form that we study where there is no obvious $Z_{2}$ symmetry associated with the period-doubling bifurcation.

Our approach to this problem is somewhat different as we use Lyapunov-Schmidt reduction to obtain the bifurcation equations, and this avoids the problems with the tail terms that occur with centre manifold reduction. We derive conditions for a Hopf bifurcation on mixed mode solution branches to exist, including the non-degeneracy condition regarding the eigenvalues crossing the unit circle. Kuznetsov and Meijer [7] take the analysis of their normal form one step further by also computing the cubic normal form coefficient for this bifurcation, which enables the direction of branching to be determined. They also study numerically the disappearance of the bifurcating tori 
via a heteroclinic bifurcation.

Iterated maps with symmetry and their bifurcations have been studied by Chossat and Golubitsky [9] and codimension two points for maps without symmetry have been studied, as discussed above. However, this work is the first study of a mode interaction involving period-doubling and symmetry-breaking bifurcations, except for the preliminary investigation in [10].

A widely used approach in studying codimension 2 problems is to use centre manifold reduction and then to derive the normal form on the centre manifold. This approach often introduces additional normal form symmetries which may exist for the normal form up to arbitrarily high order. However, the tail terms may, and often do, break this symmetry [11]. Since the tail terms are of high order, their contribution in magnitude is very small, but if they do break the normal form symmetry, then this can have a significant impact on the structure of the solutions. Period-doubling bifurcations introduce a $Z_{2}$ normal form symmetry and so the normal form equations for the type of mode interaction we are considering will have $Z_{2} \times Z_{2}$ symmetry. However, this additional $Z_{2}$ normal form symmetry may be broken by the tail terms, and so these cannot be ignored.

To avoid these problems with the tail terms of the normal form, we use a different approach which is based on Lyapunov-Schmidt reduction. A period-doubling bifurcation in an $m$-dimensional map can be reformulated as a symmetry-breaking bifurcation in a $2 m$-dimensional system of algebraic equations, where the symmetric solutions correspond to fixed points and the non-symmetric solutions are the period 2 points [12]. A Lyapunov-Schmidt reduction can then be used to reduce the system to a one-dimensional bifurcation equation. The advantage of this approach is that the Lyapunov-Schmidt process preserves the symmetry of the original system exactly [2], and so the problem of the tail terms in the normal form equations is avoided. The price that is paid for this approach is that only the fixed points and period two points are considered and so the full dynamics of the problem has been lost. However, this is sufficient for our purpose. 
We adapt this approach by taking our system of equations at a mode interaction and deriving an enlarged system with $Z_{2} \times Z_{2}$ symmetry. A Lyapunov-Schmidt reduction is then performed on this system to give two bifurcation equations which also have $Z_{2} \times Z_{2}$ symmetry, so that the results of Langford and Iooss [1] and Golubitsky and Schaeffer [2] can be applied to this problem. Since the resulting bifurcation equations are only algebraic in nature, it is not possible to infer dynamic information beyond the existence of period 2 points from the equations. Thus, we proceed to derive dynamic information regarding an invariant manifold between fixed points and conditions for the existence of a tertiary Hopf bifurcation, by taking the solutions obtained from the bifurcation equations and substituting these back into the original equations.

In Section 2, we use the method of Peckham and Kevrekidis [12] to reformulate a period-doubling bifurcation as a symmetry-breaking bifurcation and then perform a Lyapunov-Schmidt reduction at a mode interaction to derive two bifurcation equations with $Z_{2} \times Z_{2}$ symmetry. Previous results for such mode interactions [1,2] are then applied to this problem in Section 3, giving a description of the different solution branches. The existence of one-dimensional invariant manifolds is also considered. It is also shown that tertiary Hopf bifurcations may exist on mixed mode solutions in some cases and conditions for their existence are derived. In Section 4, we briefly consider the situation where the symmetry-breaking bifurcation is also period-doubling, and show that this case is very closely related to the previous case. Finally, applications to symmetricallycoupled maps and to two coupled, vertically forced pendulums are considered in Section 5.

\section{$2 \quad Z_{2} \times Z_{2}$ Symmetric Formulation of the Mode In- teraction}

Consider the following map

$$
\begin{aligned}
& X_{n+1}=F\left(X_{n}, Y_{n}, \lambda, \mu\right), \\
& Y_{n+1}=G\left(X_{n}, Y_{n}, \lambda, \mu\right),
\end{aligned}
$$


where $F, G: \mathbf{R}^{2 m} \times \mathbf{R}^{2} \rightarrow \mathbf{R}^{m}$ are smooth functions. We assume that the system (1) has $Z_{2}$ symmetry generated by the reflection $S$ which acts as

$$
S\left[\begin{array}{l}
X \\
Y
\end{array}\right]=\left[\begin{array}{r}
X \\
-Y
\end{array}\right]
$$

The subspace defined by $Y=0$ is invariant as a consequence of this symmetry. We define

$$
F_{0}(X, \lambda)=F(X, 0, \lambda, \mu)
$$

which we assume is independent of $\mu$. Thus, $\mu$ is a normal parameter for this system [13]. The dynamics in the invariant subspace is then given by

$$
X_{n+1}=F_{0}\left(X_{n}, \lambda\right) .
$$

Suppose that (2) has a branch of symmetric fixed points given by $(X(\lambda), 0)$ where $X(\lambda)$ is found by solving the fixed point equation

$$
X(\lambda)=F_{0}(X(\lambda), \lambda)
$$

Let $\lambda=\lambda_{0}$ be a period-doubling bifurcation point on this branch. Clearly, this bifurcation point is not affected by changing values of the normal parameter $\mu$. However, a symmetry-breaking bifurcation from this branch of periodic points will depend on $\mu$ and we assume that when $\mu=\mu_{0}$, a symmetry-breaking bifurcation also occurs at $\lambda=\lambda_{0}$. In this case, there is a mode interaction at $(\lambda, \mu)=\left(\lambda_{0}, \mu_{0}\right)$. We now consider two different approaches to deriving the two bifurcation equations associated with this mode interaction.

\subsection{Method 1}

A centre manifold reduction can be performed about the point $(X, Y, \lambda, \mu)=\left(X\left(\lambda_{0}\right), 0, \lambda_{0}, \mu_{0}\right)$ to reduce (1) to two dimensions. The new equations after this reduction are given by

$$
\begin{aligned}
& x_{n+1}=f\left(x_{n}, y_{n}, \alpha, \beta\right), \\
& y_{n+1}=g\left(x_{n}, y_{n}, \alpha, \beta\right),
\end{aligned}
$$


where $f, g: \mathbf{R}^{4} \rightarrow \mathbf{R}$ and

$$
\alpha=\lambda-\lambda_{0}, \quad \beta=\mu-\mu_{0} .
$$

If $m=1$ this involves only a simple change of origin given by

$$
x_{n}=X_{n}-X(\lambda), \quad y_{n}=Y_{n} .
$$

We refer to (1) as the global equations and to (3) as the local or reduced equations. Note that we are not putting these equations in normal form, and so the problems referred to in the Introduction concerning normal form symmetries do not arise here.

The equations (3) have the trivial fixed point $(x, y)=(0,0)$ for all $\alpha$ and $\beta$ and the mode interaction occurs at $(\alpha, \beta)=(0,0)$. These reduced equations inherit the $Z_{2}$ symmetry $S[14,15]$ which acts as

$$
S\left[\begin{array}{l}
x \\
y
\end{array}\right]=\left[\begin{array}{r}
x \\
-y
\end{array}\right]
$$

The symmetry again gives rise to an invariant subspace defined by $y=0$ and in this subspace the dynamics is given by

$$
x_{n+1}=f_{0}\left(x_{n}, \alpha\right)
$$

where $f_{0}(x, \alpha)=f(x, 0, \alpha, \beta)$ which does not depend on $\beta$ as it is a normal parameter. To reformulate the period-doubling bifurcation as a symmetry-breaking bifurcation we use the method of Peckham and Kevrekidis [12] and so consider a system of four equations given by

$$
\begin{aligned}
& f\left(x_{2}, y_{2}, \alpha, \beta\right)-x_{1}=0 \\
& g\left(x_{2}, y_{2}, \alpha, \beta\right)-y_{1}=0 \\
& f\left(x_{1}, y_{1}, \alpha, \beta\right)-x_{2}=0 \\
& g\left(x_{1}, y_{1}, \alpha, \beta\right)-y_{2}=0 .
\end{aligned}
$$

Solutions of these equations correspond to fixed points and period 2 points of (3). These equations inherit the symmetry of equations (3) but also have a new symmetry, and so 
the full symmetry group is $Z_{2} \times Z_{2}$ generated by $S_{1}$ and $S_{2}$ which act as

$$
S_{1}\left[\begin{array}{l}
x_{1} \\
y_{1} \\
x_{2} \\
y_{2}
\end{array}\right]=\left[\begin{array}{r}
x_{1} \\
-y_{1} \\
x_{2} \\
-y_{2}
\end{array}\right], \quad S_{2}\left[\begin{array}{l}
x_{1} \\
y_{1} \\
x_{2} \\
y_{2}
\end{array}\right]=\left[\begin{array}{l}
x_{2} \\
y_{2} \\
x_{1} \\
y_{1}
\end{array}\right] .
$$

Note that solutions of (5) which are fixed by the full symmetry group are symmetric fixed points of (3). If the $S_{1}$ symmetry is broken, this corresponds to a symmetry-breaking bifurcation of fixed points of (3) whereas breaking the $S_{2}$ symmetry corresponds to a period-doubling bifurcation of symmetric solutions of (3). A mode interaction occurs if these two types of bifurcation coincide.

To put the $S_{2}$ symmetry in more standard form, we perform a change of basis and define

$$
U=\left[\begin{array}{l}
u_{1} \\
u_{2} \\
u_{3} \\
u_{4}
\end{array}\right]=\left[\begin{array}{c}
\frac{1}{2}\left(x_{1}+x_{2}\right) \\
\frac{1}{2}\left(y_{1}+y_{2}\right) \\
\frac{1}{2}\left(x_{1}-x_{2}\right) \\
\frac{1}{2}\left(y_{1}-y_{2}\right)
\end{array}\right]
$$

The symmetries act on $U$ as

$$
S_{1}\left[\begin{array}{l}
u_{1} \\
u_{2} \\
u_{3} \\
u_{4}
\end{array}\right]=\left[\begin{array}{r}
u_{1} \\
-u_{2} \\
u_{3} \\
-u_{4}
\end{array}\right], \quad S_{2}\left[\begin{array}{l}
u_{1} \\
u_{2} \\
u_{3} \\
u_{4}
\end{array}\right]=\left[\begin{array}{r}
u_{1} \\
u_{2} \\
-u_{3} \\
-u_{4}
\end{array}\right] .
$$

In the new coordinates, equations (5) can be expressed as

$$
\begin{aligned}
& \frac{1}{2}\left[f\left(u_{1}-u_{3}, u_{2}-u_{4}, \alpha, \beta\right)+f\left(u_{1}+u_{3}, u_{2}+u_{4}, \alpha, \beta\right)\right]-u_{1}=0, \\
& \frac{1}{2}\left[g\left(u_{1}-u_{3}, u_{2}-u_{4}, \alpha, \beta\right)+g\left(u_{1}+u_{3}, u_{2}+u_{4}, \alpha, \beta\right)\right]-u_{2}=0, \\
& \frac{1}{2}\left[f\left(u_{1}-u_{3}, u_{2}-u_{4}, \alpha, \beta\right)-f\left(u_{1}+u_{3}, u_{2}+u_{4}, \alpha, \beta\right)\right]-u_{3}=0, \\
& \frac{1}{2}\left[g\left(u_{1}-u_{3}, u_{2}-u_{4}, \alpha, \beta\right)-g\left(u_{1}+u_{3}, u_{2}+u_{4}, \alpha, \beta\right)\right]-u_{4}=0,
\end{aligned}
$$

which we also express in the form

$$
\mathcal{F}(U, \alpha, \beta)-U=0
$$

where $\mathcal{F}: \mathbf{R}^{4} \times \mathbf{R}^{2} \rightarrow \mathbf{R}^{4}$.

In the two parameter plane, there is a line of period-doubling bifurcation points from the trivial symmetric fixed point to symmetric period 2 points of (3) which is defined by 
$\alpha=0$. On this path $f_{x}^{0}=-1$ where superscript zero denotes evaluation at the trivial fixed point. The reflectional symmetry of the equations (3) implies that the Jacobian evaluated at a symmetric fixed point is diagonal and so there is also a line of symmetrybreaking bifurcations defined by $g_{y}^{0}=1$, where $g_{y}^{0}$ is the other diagonal entry of the Jacobian. These two paths cross at the mode interaction point $(\alpha, \beta)=(0,0)$.

We now want to reduce the four equations (8) to a system of two equations using Lyapunov-Schmidt reduction [2] at the mode interaction. Now the Jacobian of equations (8) evaluated at the trivial fixed point is diagonal and is given by

$$
\mathcal{F}_{U}^{0}-I=\operatorname{diag}\left(f_{x}^{0}-1, g_{y}^{0}-1,-f_{x}^{0}-1,-g_{y}^{0}-1\right)
$$

Thus if $f_{x}^{0}=-1$ and $g_{y}^{0}=1$ then $\mathcal{F}_{U}^{0}-I$ has a two dimensional null space given by

$$
\operatorname{Null}\left(\mathcal{F}_{U}^{0}-I\right)=\operatorname{span}\left\{\phi_{1}, \phi_{2}\right\}
$$

where $\phi_{1}=(0,1,0,0)^{T}$ and $\phi_{2}=(0,0,1,0)^{T}$. Thus, the natural coordinates for this null space are $u_{2}$ and $u_{3}$. The corresponding left eigenvectors of $\mathcal{F}_{U}^{0}-I$ associated with the two zero eigenvalues are given by $\psi_{1}=\phi_{1}, \psi_{2}=\phi_{2}$. The bifurcation equations are thus

$$
\begin{aligned}
\psi_{1}^{T} \mathcal{F}\left(U_{0}+u_{2} \phi_{1}+u_{3} \phi_{2}+w\left(u_{2}, u_{3}, \alpha, \beta\right), \alpha, \beta\right) & =0, \\
\psi_{2}^{T} \mathcal{F}\left(U_{0}+u_{2} \phi_{1}+u_{3} \phi_{2}+w\left(u_{2}, u_{3}, \alpha, \beta\right), \alpha, \beta\right) & =0,
\end{aligned}
$$

where $U_{0}=0$ and $w\left(u_{2}, u_{3}, \alpha, \beta\right)=\left(h_{1}\left(u_{2}, u_{3}, \alpha, \beta\right), 0,0, h_{4}\left(u_{2}, u_{3}, \alpha, \beta\right)\right)^{T}$. These naturally correspond to the second and third equations. Thus, the first and last equations of (8) are used to find the low order terms of $h_{1}$ and $h_{4}$ and these are then substituted into the second and third equations to give a system of two equations in $u_{2}$ and $u_{3}$ with $Z_{2} \times Z_{2}$ symmetry

To be more precise, consider the equations

$$
\begin{aligned}
x_{n+1} & =f\left(x_{n}, y_{n}, \alpha, \beta\right) \\
& =-x_{n}-a_{2} x_{n} \alpha-a_{3} x_{n} \beta+a_{4} x_{n}^{2}+a_{5} y_{n}^{2}+a_{6} x_{n}^{3}+a_{7} x_{n} y_{n}^{2}, \\
y_{n+1} & =g\left(x_{n}, y_{n}, \alpha, \beta\right) \\
& =y_{n}\left(1+b_{1} x_{n}+b_{2} \alpha+b_{3} \beta+b_{4} x_{n}^{2}+b_{5} y_{n}^{2}\right),
\end{aligned}
$$


which have the required symmetry and satisfy the conditions that $f_{x}^{0}=-1, g_{y}^{0}=1$. Note that these equations do not have $\beta$ as a normal parameter, as we assumed previously. However, setting $a_{3}=0$ ensures that $\beta$ is a normal parameter, although we analyse these equations without making this assumption.

In $U$ coordinates, the equations (8) are given by

$$
\begin{aligned}
-\left(2+a_{2} \alpha+a_{3} \beta\right) u_{1}+a_{4}\left(u_{1}^{2}+u_{3}^{2}\right)+a_{5}\left(u_{2}^{2}+u_{4}^{2}\right) & \\
+a_{6}\left(3 u_{1} u_{3}^{2}+u_{1}^{3}\right)+a_{7}\left(u_{1} u_{2}^{2}+u_{1} u_{4}^{2}+2 u_{2} u_{3} u_{4}\right) & =0, \\
\left(b_{2} \alpha+b_{3} \beta\right) u_{2}+b_{1}\left(u_{1} u_{2}+u_{3} u_{4}\right)+b_{4}\left(u_{1}^{2} u_{2}+u_{2} u_{3}^{2}+2 u_{1} u_{3} u_{4}\right)+b_{5}\left(3 u_{2} u_{4}^{2}+u_{2}^{3}\right) & =0, \\
\left(a_{2} \alpha+a_{3} \beta\right) u_{3}-2 a_{4} u_{1} u_{3}-2 a_{5} u_{2} u_{4}-a_{6}\left(3 u_{1}^{2} u_{3}+u_{3}^{3}\right)-a_{7}\left(u_{2}^{2} u_{3}+u_{3} u_{4}^{2}+2 u_{1} u_{2} u_{4}\right) & =0, \\
-\left(2+b_{2} \alpha+b_{3} \beta\right) u_{4}-b_{1}\left(u_{2} u_{3}+u_{1} u_{4}\right) & \\
-b_{4}\left(u_{1}^{2} u_{4}+u_{3}^{2} u_{4}+2 u_{1} u_{2} u_{3}\right)-b_{5}\left(3 u_{2}^{2} u_{4}+u_{4}^{3}\right) & =0 .
\end{aligned}
$$

The functions $h_{1}$ and $h_{4}$ are then found to lowest order as

$$
\begin{aligned}
& u_{1}=h_{1}\left(u_{2}, u_{3}, \alpha, \beta\right)=\frac{1}{2}\left(a_{5} u_{2}^{2}+a_{4} u_{3}^{2}\right), \\
& u_{4}=h_{4}\left(u_{2}, u_{3}, \alpha, \beta\right)=-\frac{1}{2} b_{1} u_{2} u_{3} .
\end{aligned}
$$

Substituting these into the second and third equations leads to the two bifurcation equations which are given to cubic order by

$$
\begin{aligned}
u\left[A u^{2}+B v^{2}+a_{2} \alpha+a_{3} \beta\right] & =0 \\
v\left[C u^{2}+D v^{2}+b_{2} \alpha+b_{3} \beta\right] & =0
\end{aligned}
$$

where $u=u_{3}, v=u_{2}$ and

$$
\begin{aligned}
A & =-\left(a_{4}^{2}+a_{6}\right), & B & =a_{5} b_{1}-a_{4} a_{5}-a_{7}, \\
C & =\frac{1}{2}\left(-b_{1}^{2}+2 b_{4}+a_{4} b_{1}\right), & D & =\frac{1}{2}\left(a_{5} b_{1}+2 b_{5}\right) .
\end{aligned}
$$

Clearly, these equations have $Z_{2} \times Z_{2}$ symmetry as anticipated. We note that any higher order terms in equations (9) will not change the coefficients in the bifurcation equations (12). 


\subsection{Method 2}

An alternative approach is to apply the method of Peckham and Kevrekidis [12] to the original system of equations (1) and then apply Lyapunov-Schmidt reduction to obtain the bifurcation equations (12) directly. The advantage of this approach is that the coefficients of the bifurcation equations (12) are expressed directly in terms of the functions $F$ and $G$ that define the original map (1).

At the mode interaction point $(\lambda, \mu)=\left(\lambda_{0}, \mu_{0}\right), F_{X}^{0}$ has a simple eigenvalue of -1 corresponding to the period-doubling bifurcation and $G_{Y}^{0}$ has a simple eigenvalue of +1 corresponding to the symmetry-breaking bifurcation, where superscript zero denotes evaluation at the fixed point $(X, Y, \lambda, \mu)=\left(X\left(\lambda_{0}\right), 0, \lambda_{0}, \mu_{0}\right)$. If $\phi_{1}$ and $\psi_{1}$ are the right and left eigenvectors of $F_{X}^{0}$ and $\phi_{2}$ and $\psi_{2}$ are the right and left eigenvectors of $G_{Y}^{0}$, then

$$
\begin{array}{ll}
F_{X}^{0} \phi_{1}=-\phi_{1}, & \psi_{1}^{T} F_{X}^{0}=-\psi_{1}^{T}, \\
G_{Y}^{0} \phi_{2}=\phi_{2}, & \psi_{2}^{T} G_{Y}^{0}=\psi_{2}^{T} .
\end{array}
$$

To apply the method of Peckham and Kevrekidis, we rewrite equations (3) as the algebraic equations

$$
\begin{aligned}
& F\left(X_{2}, Y_{2}, \lambda, \mu\right)-X_{1}=0, \\
& G\left(X_{2}, Y_{2}, \lambda, \mu\right)-Y_{1}=0, \\
& F\left(X_{1}, Y_{1}, \lambda, \mu\right)-X_{2}=0, \\
& G\left(X_{1}, Y_{1}, \lambda, \mu\right)-Y_{2}=0 .
\end{aligned}
$$

These equations again have solutions corresponding to fixed points and period 2 points of (3). They also have $Z_{2} \times Z_{2}$ symmetry generated by $S_{1}$ and $S_{2}$ which act on $\left(X_{1}, Y_{1}, X_{2}, Y_{2}\right)$ in a similar way to (6). A change of coordinates could be used as in the previous section, but it is as easy to do the Lyapunov-Schmidt reduction directly without this change.

The Jacobian of equations (15) is given by

$$
\left[\begin{array}{cccc}
-I & 0 & F_{X}^{0} & 0 \\
0 & -I & 0 & G_{Y}^{0} \\
F_{X}^{0} & 0 & -I & 0 \\
0 & G_{Y}^{0} & 0 & -I
\end{array}\right]
$$


This matrix has a two-dimensional null space with right nullvectors $\Phi_{1}, \Phi_{2}$ and left nullvectors $\Psi_{1}, \Psi_{2}$ which are given by

$$
\Phi_{1}=\left[\begin{array}{c}
\phi_{1} \\
0 \\
-\phi_{1} \\
0
\end{array}\right], \quad \Phi_{2}=\left[\begin{array}{c}
0 \\
\phi_{2} \\
0 \\
\phi_{2}
\end{array}\right], \quad \Psi_{1}=\left[\begin{array}{c}
\psi_{1} \\
0 \\
-\psi_{1} \\
0
\end{array}\right], \quad \Psi_{2}=\left[\begin{array}{c}
0 \\
\psi_{2} \\
0 \\
\psi_{2}
\end{array}\right]
$$

Applying Lyapunov-Schmidt reduction to this system gives two bifurcation equations of the form (12). We do not go through the details as the procedure is standard. We simply note that the resulting coefficients of equations (12) are given by

$$
\begin{gathered}
A=\psi_{1}^{T}\left(F_{X X}^{0} \phi_{1} w_{3}+\frac{1}{3} F_{X X X}^{0} \phi_{1} \phi_{1} \phi_{1}\right), \\
B=\psi_{1}^{T}\left(F_{X X}^{0} \phi_{1} w_{4}+2 F_{Y Y}^{0} \phi_{2} w_{5}+F_{X Y Y}^{0} \phi_{1} \phi_{2} \phi_{2}\right), \\
C=\psi_{2}^{T}\left(G_{X Y}^{0} \phi_{2} w_{3}+2 G_{X Y}^{0} \phi_{1} w_{5}+G_{X X Y}^{0} \phi_{1} \phi_{1} \phi_{2}\right), \\
D=\psi_{2}^{T}\left(G_{X Y}^{0} \phi_{2} w_{4}+\frac{1}{3} G_{Y Y Y}^{0} \phi_{2} \phi_{2} \phi_{2}\right), \\
a_{2}=2 \psi_{1}^{T}\left(F_{X X}^{0} \phi_{1} w_{1}+F_{X \lambda}^{0} \phi_{1}\right), \quad a_{3}=2 \psi_{1}^{T}\left(F_{X X}^{0} \phi_{1} w_{2}+F_{X \mu}^{0} \phi_{1}\right), \\
b_{2}=2 \psi_{2}^{T}\left(G_{X Y}^{0} \phi_{2} w_{1}+G_{Y \lambda}^{0} \phi_{2}\right), \quad b_{3}=2 \psi_{2}^{T}\left(G_{X Y}^{0} \phi_{2} w_{2}+G_{Y \mu}^{0} \phi_{2}\right),
\end{gathered}
$$

where the functions $w_{i}, i=1, \ldots, 5$ are found as the unique solution of the equations

$$
\begin{array}{rlrl}
\left(F_{X}^{0}-I\right) w_{1}+F_{\lambda}^{0} & =0, & \left(F_{X}^{0}-I\right) w_{2}+F_{\mu}^{0}=0, \\
\left(F_{X}^{0}-I\right) w_{3}+F_{X X}^{0} \phi_{1} \phi_{1} & =0, & \left(F_{X}^{0}-I\right) w_{4}+F_{Y Y}^{0} \phi_{2} \phi_{2}=0, \\
\left(G_{Y}^{0}+I\right) w_{5}+G_{X Y}^{0} \phi_{1} \phi_{2} & =0 . & &
\end{array}
$$

\section{Solutions in the Neighbourhood of the Mode In- teraction}

Having formulated the mode interaction problem as one which has $Z_{2} \times Z_{2}$ symmetry, we can now apply previous results for such mode interactions to this problem $[1,2]$.

\subsection{Non-degeneracy conditions}

The mode interaction problem is nondegenerate [2] if all of the following conditions are satisfied:

$$
A=-\left(a_{4}^{2}+a_{6}\right) \neq 0, \quad D=\frac{1}{2}\left(a_{5} b_{1}+2 b_{5}\right) \neq 0, \quad a_{2} \neq 0, \quad b_{2} \neq 0,
$$




$$
\begin{aligned}
A D-B C & =-\frac{1}{2}\left(a_{4}^{2}+a_{6}\right)\left(a_{5} b_{1}+2 b_{5}\right)-\frac{1}{2}\left(a_{5} b_{1}-a_{4} a_{5}-a_{7}\right)\left(-b_{1}^{2}+2 b_{4}+a_{4} b_{1}\right) \neq 0 \\
b_{2} A-a_{2} C & =-\frac{1}{2}\left[2 b_{2}\left(a_{4}^{2}+a_{6}\right)+a_{2}\left(-b_{1}^{2}+2 b_{4}+a_{4} b_{1}\right)\right] \neq 0 \\
b_{2} B-a_{2} D & =\frac{1}{2}\left[2 b_{2}\left(a_{5} b_{1}-a_{4} a_{5}-a_{7}\right)-a_{2}\left(a_{5} b_{1}+2 b_{5}\right)\right] \neq 0 .
\end{aligned}
$$

The second parameter $\beta$ is an unfolding parameter for the mode interaction if [2]

$$
b_{2} a_{3}-a_{2} b_{3} \neq 0
$$

If $\beta$ is a normal parameter, then $a_{3}=0$ and so this condition then reduces to $a_{2} b_{3} \neq 0$.

We introduce some constants to help keep the equations simple, which are given by

$$
\begin{array}{rlrl}
D_{0} & =a_{2} b_{3}-a_{3} b_{2}, & \Delta & =A D-B C, \\
D_{1}^{\alpha} & =a_{2} C-b_{2} A, & D_{1}^{\beta} & =a_{3} C-b_{3} A, \\
D_{2}^{\alpha} & =a_{2} D-b_{2} B, & D_{2}^{\beta}=a_{3} D-b_{3} B .
\end{array}
$$

\subsection{Solution branches}

We now consider the different solution branches of the bifurcation equations (12).

1. The trivial solution is given by $u=v=0$.

2. The primary $u$-branch of solutions is given by

$$
u^{2}=-\frac{a_{2} \alpha+a_{3} \beta}{A}, \quad v=0
$$

3. The primary $v$-branch of solutions is given by

$$
u=0, \quad v^{2}=-\frac{b_{2} \alpha+b_{3} \beta}{D} .
$$

4. Mixed mode solutions can be found by solving equations (12) simultaneously giving

$$
u^{2}=\frac{-D_{2}^{\alpha} \alpha-D_{2}^{\beta} \beta}{\Delta}, \quad v^{2}=\frac{D_{1}^{\alpha} \alpha+D_{1}^{\beta} \beta}{\Delta},
$$

where $D_{1}^{\alpha}, D_{1}^{\beta}, D_{2}^{\alpha}, D_{2}^{\beta}$ and $\Delta$ are given in (18). 
From the solutions in terms of $u=u_{3}$ and $v=u_{2}$ the functions $u_{1}$ and $u_{4}$ can be obtained from (10) and (11). Then, by using the inverse of the transformation (7), the solution branches can be expressed in terms of $x_{1}, x_{2}, y_{1}$ and $y_{2}$.

1. Clearly the trivial solution $u=v=0$ corresponds to $x_{1}=x_{2}=y_{1}=y_{2}=0$ and exists for all values of $\alpha$ and $\beta$.

2. On the primary $u$-branch (19), we have $u_{1}=\frac{1}{2} a_{4} u^{2}, u_{4}=0$. With $u=u_{3}$ and $v=u_{2}=0$ the solutions for the primary $u$-branch in $(x, y)$-coordinates are given by

$$
\begin{gathered}
x_{1}^{2}=-\frac{\left(a_{2} \alpha+a_{3} \beta\right)}{A}+O\left((\alpha, \beta)^{\frac{3}{2}}\right), \quad x_{2}^{2}=-\frac{\left(a_{2} \alpha+a_{3} \beta\right)}{A}+O\left((\alpha, \beta)^{\frac{3}{2}}\right), \\
y_{1}=y_{2}=0 .
\end{gathered}
$$

Note that $x_{1}^{2}$ and $x_{2}^{2}$ are the same to first order in $\alpha$ and $\beta$, but they are different at higher order. Also $x_{1}$ and $x_{2}$ will have opposite signs as they are period two points. Thus these solutions are symmetric period 2 points. This branch bifurcates from the trivial solution at $\alpha=-\frac{a_{3}}{a_{2}} \beta$ and is supercritical if $a_{2} A<0$ or subcritical if $a_{2} A>0$.

Recall that $\beta$ is a normal parameter if $a_{3}=0$. In this case, it is clear that these solutions do not depend on $\beta$ and that they bifurcate from the line $\alpha=0$.

3. On the primary $v$-branch (20), we have $u_{1}=\frac{1}{2} a_{5} v^{2}, u_{4}=0$. With $u=u_{3}=0$ and $v=u_{2}$ the solutions for the primary $v$-branch in $(x, y)$-coordinates are given by

$$
x_{1}=x_{2}=-\frac{a_{5}\left(b_{2} \alpha+b_{3} \beta\right)}{2 D}, \quad y_{1}^{2}=y_{2}^{2}=-\frac{b_{2} \alpha+b_{3} \beta}{D} .
$$

Here $y_{1}=y_{2}$ since it is a branch of fixed points. Thus these solutions are nonsymmetric fixed points. This branch bifurcates from the trivial solution at $\alpha=$ $-\frac{b_{3}}{b_{2}} \beta$ and is supercritical if $b_{2} D<0$ or subcritical if $b_{2} D>0$.

The non-degeneracy condition (17) ensures that the two paths of primary bifurcation points are distinct, and so cross only at the origin. 
4. For the mixed mode solutions we have

$$
u_{1}=\frac{1}{2}\left(a_{4} u^{2}+a_{5} v^{2}\right), \quad u_{4}=-\frac{1}{2} b_{1} u v .
$$

With $u=u_{3}$ and $v=u_{2}$ the mixed mode solutions in $(x, y)$-coordinates are given by

$$
\begin{array}{ll}
x_{1}^{2}=\frac{-D_{2}^{\alpha} \alpha-D_{2}^{\beta} \beta}{\Delta}+O\left((\alpha, \beta)^{\frac{3}{2}}\right), & x_{2}^{2}=\frac{-D_{2}^{\alpha} \alpha-D_{2}^{\beta} \beta}{\Delta}+O\left((\alpha, \beta)^{\frac{3}{2}}\right), \\
y_{1}^{2}=\frac{D_{1}^{\alpha} \alpha+D_{1}^{\beta} \beta}{\Delta}+O\left((\alpha, \beta)^{\frac{3}{2}}\right), & y_{2}^{2}=\frac{D_{1}^{\alpha} \alpha+D_{1}^{\beta} \beta}{\Delta}+O\left((\alpha, \beta)^{\frac{3}{2}}\right) .
\end{array}
$$

Note that again $x_{1}^{2}$ and $x_{2}^{2}$ are the same to first order in $\alpha$ and $\beta$ but they are different at higher order. Also, $x_{1}$ and $x_{2}$ will have opposite signs as they are period two points. Similarly, $y_{1}^{2}$ and $y_{2}^{2}$ are different at higher order and $y_{1}$ and $y_{2}$ have the same signs with a second conjugate solution where $y_{1}$ and $y_{2}$ both change sign. Thus, these solutions are non-symmetric period 2 points.

Setting $y_{1}^{2}=y_{2}^{2}=0$ implies that the secondary bifurcation on the branch of symmetric period 2 points occurs at

$$
\alpha=\alpha_{x}=-\frac{D_{1}^{\beta}}{D_{1}^{\alpha}} \beta \quad \text { when } \quad \beta D_{0} D_{1}^{\alpha}<0 .
$$

The inequality is obtained by substituting $\alpha=\alpha_{x}$ into the first or second equation in (23). This bifurcation is a symmetry-breaking bifurcation of period 2 points and is supercritical if $\Delta D_{1}^{\alpha}>0$ or subcritical if $\Delta D_{1}^{\alpha}<0$.

Similarly, setting $x_{1}^{2}=x_{2}^{2}=0$ implies that the secondary bifurcation on the branch of non-symmetric fixed points occurs at

$$
\alpha=\alpha_{y}=-\frac{D_{2}^{\beta}}{D_{2}^{\alpha}} \beta \quad \text { when } \quad \beta D_{0} D_{2}^{\alpha}<0 .
$$

The inequality is obtained by substituting $\alpha=\alpha_{y}$ into the third or fourth equation in (23). This bifurcation is a period-doubling bifurcation and is supercritical if $\Delta D_{2}^{\alpha}<0$ or subcritical if $\Delta D_{2}^{\alpha}>0$. 
Using (24) and (25), equations (23) can also be written in the form

$$
\begin{array}{ll}
x_{1}^{2}=\frac{-\left(\alpha-\alpha_{y}\right) D_{2}^{\alpha}}{\Delta}+O\left((\alpha, \beta)^{\frac{3}{2}}\right), & x_{2}^{2}=\frac{-\left(\alpha-\alpha_{y}\right) D_{2}^{\alpha}}{\Delta}+O\left((\alpha, \beta)^{\frac{3}{2}}\right), \\
y_{1}^{2}=\frac{\left(\alpha-\alpha_{x}\right) D_{1}^{\alpha}}{\Delta}+O\left((\alpha, \beta)^{\frac{3}{2}}\right), & y_{2}^{2}=\frac{\left(\alpha-\alpha_{x}\right) D_{1}^{\alpha}}{\Delta}+O\left((\alpha, \beta)^{\frac{3}{2}}\right) .
\end{array}
$$

The inequalities in (24) and (25) imply that the two secondary bifurcations occur for the same sign of $\beta$ if and only if $D_{1}^{\alpha} D_{2}^{\alpha}>0$ and for opposite signs of $\beta$ if and only if $D_{1}^{\alpha} D_{2}^{\alpha}<0$. Thus, mixed mode solutions only exist for one sign of $\beta$ if $D_{1}^{\alpha} D_{2}^{\alpha}>0$ but they exist for both signs of $\beta$ if $D_{1}^{\alpha} D_{2}^{\alpha}<0$.

\subsection{One-dimensional invariant manifolds}

The bifurcation equations (12) are algebraic equations and hence there is no dynamics associated with them. The related vector field equations given by

$$
\begin{aligned}
& \dot{u}=u\left[A u^{2}+B v^{2}+a_{2} \alpha+a_{3} \beta\right], \\
& \dot{v}=v\left[C u^{2}+D v^{2}+b_{2} \alpha+b_{3} \beta\right],
\end{aligned}
$$

are often considered $[1,2]$ and for these equations both the $u$ and $v$ axes are invariant under the flow. We now consider whether there are similar one-dimensional invariant manifolds for our discrete problem.

Due to the symmetry (4), the $x$-axis in (3) corresponds to a fixed point space and is therefore invariant under iteration. However, as the second symmetry in the bifurcation equations is not a symmetry of the original problem, the $y$-axis is not invariant in general. However, we now show that if $a_{5} \neq 0$ then there is a one-dimensional manifold passing through the trivial fixed point which is invariant and is tangent to the $y$-axis at the origin. We also show that if a pair of non-symmetric fixed points exist for particular small values of $\alpha$ and $\beta$, then this invariant manifold passes through these points, giving rise to heteroclinic connections between the trivial fixed point and these non-symmetric fixed points.

Suppose that the invariant manifold is given by

$$
x=h(y)
$$


We require that it must pass through the origin and be invariant under reflection in $y$. Thus, we can write

$$
h(y)=d_{1} y^{2}+d_{2} y^{4}+O\left(y^{6}\right) .
$$

Clearly, this curve is tangent to the $y$-axis at the origin. We also want it to be flow invariant. For given values $\left(x_{0}, y_{0}\right)$, let $x_{1}=f\left(x_{0}, y_{0}, \alpha, \beta\right)$ and $y_{1}=g\left(x_{0}, y_{0}, \alpha, \beta\right)$. The curve is then invariant if $x_{0}=h\left(y_{0}\right)$ implies that $x_{1}=h\left(y_{1}\right)$ which is equivalent to

$$
f\left(h\left(y_{0}\right), y_{0}, \alpha, \beta\right)=h\left(g\left(h\left(y_{0}\right), y_{0}, \alpha, \beta\right)\right)
$$

Equation (28) is invariant under the transformation $y_{0} \rightarrow-y_{0}$ and $y_{0}=0$ is a solution of this equation. Substituting for $h(y)$ using (27) and equating powers of $y^{2}$, we can calculate the coefficients $d_{i}, i=1,2$. We consider only the lowest order term and equating powers of $y_{0}^{2}$ gives an equation which can be solved for $d_{1}$ giving

$$
d_{1}=\frac{a_{5}}{2+\left(a_{2}+2 b_{2}\right) \alpha+\left(a_{3}+2 b_{3}\right) \beta+\left(b_{2} \alpha+b_{3} \beta\right)^{2}}=\frac{1}{2} a_{5}+O(\alpha, \beta) .
$$

The iteration on the invariant manifold is given by the single equation

$$
y_{n+1}=g\left(h\left(y_{n}\right), y_{n}, \alpha, \beta\right)
$$

and for any value $y_{n+1}$ we have, using (28), that

$$
x_{n+1}=h\left(y_{n+1}\right)=f\left(h\left(y_{n}\right), y_{n}, \alpha, \beta\right)=f\left(x_{n}, y_{n}, \alpha, \beta\right) .
$$

If a non-symmetric fixed point exists and lies on this manifold, then it must be a solution of the equation

$$
y=g(h(y), y, \alpha, \beta)
$$

One solution of this equation is $y=0$. A second solution of this equation is sufficient to establish the existence of a heteroclinic connection between fixed points.

To find non-trivial solutions of (31) we divide it by $y$ giving

$$
\tilde{g}\left(y^{2}, \alpha, \beta\right)=\frac{g(h(y), y, \alpha, \beta)-y}{y}=b_{2} \alpha+b_{3} \beta+\left(b_{5}+d_{1} b_{1}\right) y^{2}+O\left(y^{4}\right)=0 .
$$


This equation has one solution $y^{2}=\alpha=\beta=0$ and so, by the Implicit Function Theorem, it can be solved for $y^{2}$ as a function of $\alpha$ and $\beta$ if $\tilde{g}_{y^{2}}(0,0,0) \neq 0$. Substituting for $d_{1}$ from (29) gives

$$
\tilde{g}_{y^{2}}(0,0,0)=b_{5}+\frac{1}{2} a_{5} b_{1}
$$

Now $b_{5}+\frac{1}{2} a_{5} b_{1}=D \neq 0$ according to the non-degeneracy conditions (16). Thus, (32) can be solved uniquely for $y^{2}=y^{2}(\alpha, \beta)$ with $y^{2}(0,0)=0$. This implies that (31) has two non-symmetric fixed points which collapse down to the trivial solution of the mode interaction as $\alpha, \beta \rightarrow 0$. Hence, these must be the non-symmetric fixed points that arise from the mode interaction.

Substituting for $d_{1}$ from (29) into (32) and solving for $y^{2}$ gives

$$
y^{2}=-\frac{b_{2} \alpha+b_{3} \beta}{D}+O\left((\alpha, \beta)^{2}\right)
$$

This is the same as the solution (22) obtained from the bifurcation equation to first order.

The constant term in the Taylor expansion of $d_{1}$ in terms of $\alpha$ and $\beta$ is given by $\frac{1}{2} a_{5}$. Thus, to lowest order, the invariant manifold (27) becomes

$$
x=h(y)=\left(\frac{1}{2} a_{5}+O(\alpha, \beta)\right) y^{2}+O\left(y^{4}\right) .
$$

Substituting for $y^{2}$ from the non-trivial fixed point given by (33) gives

$$
x=-\frac{a_{5}\left(b_{2} \alpha+b_{3} \beta\right)}{2 D}+O\left((\alpha, \beta)^{2}\right) .
$$

This solution is also in agreement with (22).

The iteration on the invariant curve is given by (30) and substituting $x=h(y)$ given by (34) into $g$ defined by (9) gives the iteration

$$
y_{n+1}=y_{n}\left[1+b_{2} \alpha+b_{3} \beta+\left(\frac{1}{2}\left(a_{5} b_{1}+2 b_{5}\right)+O(\alpha, \beta)\right) y_{n}^{2}\right]+O\left(y_{n}^{5}\right)
$$

Including more higher order terms in $f$ and $g$ defined by (9) will have no effect on these low order terms, and so this result holds for more general functions $f$ and $g$ as well. 


\subsection{Tertiary Hopf bifurcations}

The vector field equations with $Z_{2} \times Z_{2}$ symmetry (26) have a tertiary Hopf bifurcation on the mixed mode branch in some cases $[1,16]$. In this section we show that a tertiary Hopf (or Neimark-Sacker) bifurcation may occur on mixed mode solutions for the perioddoubling/symmetry-breaking mode interaction also.

To derive conditions for a possible tertiary Hopf bifurcation on the period 2 mixed mode solutions in our discrete problem, we consider the equations associated with two iterations of the reduced equations (3) which are given by

$$
\begin{aligned}
& x_{n+2}=F\left(x_{n}, y_{n}, \alpha, \beta\right), \\
& y_{n+2}=G\left(x_{n}, y_{n}, \alpha, \beta\right),
\end{aligned}
$$

where

$$
\begin{aligned}
& F(x, y, \alpha, \beta)=f(f(x, y, \alpha, \beta), g(x, y, \alpha, \beta), \alpha, \beta), \\
& G(x, y, \alpha, \beta)=g(f(x, y, \alpha, \beta), g(x, y, \alpha, \beta), \alpha, \beta) .
\end{aligned}
$$

Suppose that the reduced equations (3) have a pair of non-symmetric period 2 points $\left(x_{(1)}, y_{(1)}\right)$ and $\left(x_{(2)}, y_{(2)}\right)$ on a mixed mode branch. Let $J_{F, G}^{1}$ denote the Jacobian of equations (36) evaluated at $\left(x_{(1)}, y_{(1)}, \alpha, \beta\right)$. Then the eigenvalues $\sigma_{1,2}$ of this matrix are given by $[17]$

$$
\sigma_{1,2}=\frac{1}{2}\left(\operatorname{tr}\left(J_{F, G}^{1}\right) \pm \sqrt{\operatorname{tr}\left(J_{F, G}^{1}\right)^{2}-4 \operatorname{det}\left(J_{F, G}^{1}\right)}\right) .
$$

Clearly, the eigenvalues will be complex if

$$
\operatorname{tr}\left(J_{F, G}^{1}\right)^{2}-4 \operatorname{det}\left(J_{F, G}^{1}\right)<0
$$

If the Jacobian has two complex conjugate eigenvalues $\sigma_{1}$ and $\sigma_{2}=\bar{\sigma}_{1}$ then Hopf bifurcation will occur when $\left|\sigma_{1}\right|=\left|\sigma_{2}\right|=1$. Now $\operatorname{det}\left(J_{F, G}^{1}\right)$ is equal to the product of the eigenvalues and so the first condition for a Hopf bifurcation is [17]

$$
\operatorname{det}\left(J_{F, G}^{1}\right)=1
$$


Using this information, inequality (37) becomes

$$
-2<\operatorname{tr}\left(J_{F, G}^{1}\right)<2
$$

To use these conditions we require the determinant and trace of the Jacobian of equations (36). For the determinant, we note that

$$
J_{F, G}\left(x_{(1)}, y_{(1)}, \alpha, \beta\right)=J_{f, g}\left(x_{(2)}, y_{(2)}, \alpha, \beta\right) J_{f, g}\left(x_{(1)}, y_{(1)}, \alpha, \beta\right)
$$

where $J_{f, g}$ is the Jacobian of the reduced equations (3), and so

$$
\operatorname{det}\left(J_{F, G}^{1}\right)=\operatorname{det}\left(J_{f, g}^{2}\right) \operatorname{det}\left(J_{f, g}^{1}\right),
$$

where $J_{f, g}^{i}=J_{f, g}\left(x_{(i)}, y_{(i)}, \alpha, \beta\right)$ for $i=1,2$. The Jacobian of the particular equations (9) is given by

$$
J_{f, g}(x, y, \alpha, \beta)=\left[\begin{array}{cc}
-1-a_{2} \alpha-a_{3} \beta+2 a_{4} x & 2 y\left(a_{5}+a_{7} x\right) \\
+3 a_{6} x^{2}+a_{7} y^{2} & \\
y\left(b_{1}+2 b_{4} x\right) & 1+b_{1} x+b_{2} \alpha+b_{3} \beta \\
& +b_{4} x^{2}+3 b_{5} y^{2}
\end{array}\right] .
$$

Evaluating this matrix at the first of the period 2 points gives

$$
J_{f, g}\left(x_{(1)}, y_{(1)}, \alpha, \beta\right)=\left[\begin{array}{cc}
\frac{x_{(2)}-a_{5} y_{(1)}^{2}}{x_{(1)}}+a_{4} x_{(1)}+2 a_{6} x_{(1)}^{2} & 2 y_{(1)}\left(a_{5}+a_{7} x_{(1)}\right) \\
y_{(1)}\left(b_{1}+2 b_{4} x_{(1)}\right) & \frac{y_{(2)}}{y_{(1)}}+2 b_{5} y_{(1)}^{2}
\end{array}\right],
$$

with a similar form for the Jacobian evaluated at the second period 2 point.

Using the inverse of the transformation (7) and relations (10) and (11), the determinant can be expressed in terms of powers of $u=u_{3}$ and $v=u_{2}$ as

$$
\operatorname{det}\left(J_{F, G}^{1}\right)=1+4\left[k_{1}(\alpha, \beta) u^{2}+k_{2}(\alpha, \beta) v^{2}\right]+O\left((u, v)^{4}\right),
$$

where

$$
k_{1}(\alpha, \beta)=A+O(\alpha, \beta), \quad k_{2}(\alpha, \beta)=D+O(\alpha, \beta),
$$

and $A$ and $D$ are given by (13). With this form of the determinant, we can now solve (38) for $v^{2}$ in terms of $u^{2}$, giving

$$
v^{2}=-\frac{k_{1}(\alpha, \beta)}{k_{2}(\alpha, \beta)} u^{2}+O\left(u^{4}\right)=\left(-\frac{A}{D}+O(\alpha, \beta)\right) u^{2}+O\left(u^{4}\right) .
$$


Clearly, for $v$ to have real solutions for small $u, \alpha$ and $\beta$, we require $A D<0$.

To calculate the trace is more laborious as there is no simple formula for the trace of a product of matrices. Thus, we find the product of the Jacobian matrices $J_{f, g}^{2}$ and $J_{f, g}^{1}$ and then find the trace of the resultant matrix giving

$$
\operatorname{tr}\left(J_{F, G}^{1}\right)=2+4\left[k_{1}(\alpha, \beta) u^{2}+k_{2}(\alpha, \beta) v^{2}\right]+O\left((u, v)^{4}\right)
$$

Substituting for $v^{2}$ from (41) eliminates the quadratic terms in this equation, and so fourth order terms are required in order to determine conditions for the right hand inequality in (39) to hold. Repeating this process but including fifth order terms in the equations and fourth order terms in the determinant and trace, we find that the trace as a function of $u$ is then given by

$$
\operatorname{tr}\left(J_{F, G}^{1}\right)=2+\left(\frac{16 A \Delta}{D}+O(\alpha, \beta)\right) u^{4}+O\left(u^{6}\right)
$$

For the existence of a Hopf bifurcation, the trace should be less than 2 by (39), and since $A D<0$ this requires that $\Delta>0$.

Substituting the mixed mode solutions (21) into (40) implies that the Hopf bifurcation occurs when

$$
\alpha=\alpha_{h}=-\frac{D_{4}}{D_{3}} \beta
$$

where

$$
D_{3}=D D_{1}^{\alpha}-A D_{2}^{\alpha}, \quad D_{4}=D D_{1}^{\beta}-A D_{2}^{\beta} .
$$

Substituting $\alpha=-\frac{D_{4}}{D_{3}} \beta$ in the mixed mode solutions (23) gives

$$
\begin{gathered}
x_{1}^{2}=-\frac{D_{0} D}{D_{3}} \beta+O\left((\alpha, \beta)^{\frac{3}{2}}\right), \quad x_{2}^{2}=-\frac{D_{0} D}{D_{3}} \beta+O\left((\alpha, \beta)^{\frac{3}{2}}\right), \\
y_{1}^{2}=y_{2}^{2}=\frac{D_{0} A}{D_{3}} \beta+O\left((\alpha, \beta)^{\frac{3}{2}}\right) .
\end{gathered}
$$

If $D_{1}^{\alpha} D_{2}^{\alpha}>0$ then according to (24) and (25) mixed mode solutions only exist if $\beta D_{0} D_{1}^{\alpha}<0$ or equivalently if $\beta D_{0} D_{2}^{\alpha}<0$. Since $A D<0$, it is then easy to verify that $\beta A D_{0} D_{3}>0$ and so $\beta D D_{0} D_{3}<0$. Therefore Hopf bifurcation only occurs when $\beta A D_{0} D_{3}>0$. For $D_{1}^{\alpha} D_{2}^{\alpha}<0$ the mixed mode solutions exist for both signs of $\beta$ but the 
Hopf bifurcation always exists for only one sign of $\beta$ which can be determined from the condition $\beta A D_{0} D_{3}>0$. Thus, in both cases, the tertiary Hopf bifurcation exists only for one sign of $\beta$ which can be determined from the condition $\beta A D_{0} D_{3}>0$.

There is also a non-degeneracy condition associated with Hopf bifurcation, namely the requirement that the complex eigenvalues actually cross the unit circle. In particular a nonzero derivative of $\left|\sigma_{1,2}\right|^{2}$ with respect to $\alpha$ at the Hopf bifurcation point ensures that the eigenvalues cross the unit circle, where

$$
\left|\sigma_{1,2}\right|^{2}=\operatorname{det}\left(J_{F, G}^{1}\right)
$$

Differentiating (40) with respect to $\alpha$, it can be shown that

$$
\frac{d\left|\sigma_{1,2}\right|^{2}}{d \alpha}=\frac{4\left(D D_{1}^{\alpha}-A D_{2}^{\alpha}\right)}{\Delta}+O(\alpha, \beta)
$$

Therefore $D D_{1}^{\alpha}-A D_{2}^{\alpha} \neq 0$ guarantees that complex conjugate eigenvalues cross the unit circle for $\alpha$ and $\beta$ sufficiently small.

We summarise these results as follows.

\section{Theorem 3.1}

A tertiary Hopf bifurcation from period 2 mixed mode solutions exists in the discrete dynamical system (9) if

$$
A D<0, \quad \Delta>0, \quad D D_{1}^{\alpha}-A D_{2}^{\alpha} \neq 0 .
$$

It occurs at

$$
\alpha=-\frac{D_{4}}{D_{3}} \beta \quad \text { when } \quad \beta A D_{0} D_{3}>0
$$

\section{Remark 3.2}

According to Arrowsmith and Place [18], in a small neighbourhood of the origin, the map

(3) can be approximated by a two-dimensional autonomous vector field where equilibria of the vector field correspond to fixed points of the map and periodic orbits of the vector field correspond to invariant circles of the map. Thus, the existence of a tertiary Hopf bifurcation for the map would be expected as there is a tertiary Hopf bifurcation in the 


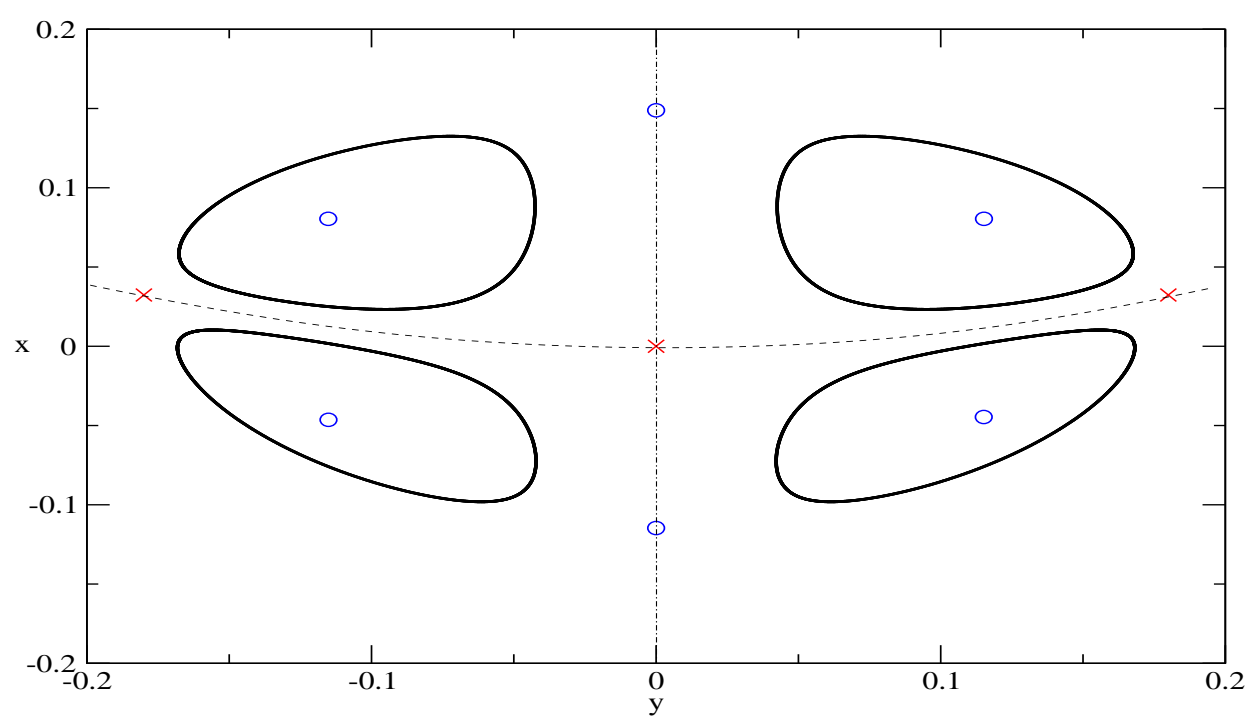

Figure 1: Phase portrait for $\alpha=0.0683$ and $\beta=-0.1 . \times-$ fixed points, o - period 2 points.

vector field but this correspondence does not provide the detailed conditions for this Hopf bifurcation to occur. However, we have shown that the conditions for this Hopf bifurcation in discrete dynamical systems are in fact the same as for the related vector field equations (26) $[1,16]$.

\section{Example 3.3}

For the reduced equations (9) we take $a_{2}=1, a_{3}=0, a_{4}=2, a_{5}=2, a_{6}=0, a_{7}=0$, $b_{1}=b_{2}=b_{3}=1, b_{4}=5, b_{5}=1$. We also include two higher order terms involving $x$ in the second equation to make the Hopf bifurcation nondegenerate. The reduced equations then become

$$
\begin{aligned}
& x_{n+1}=-x_{n}-x_{n} \alpha+2 x_{n}^{2}+2 y_{n}^{2}, \\
& y_{n+1}=y_{n}\left[1+x_{n}+\alpha+\beta+5 x_{n}^{2}+y_{n}^{2}-10 \alpha x_{n}+5 \beta x_{n}\right] .
\end{aligned}
$$

The bifurcation equations for this system are

$$
\begin{gathered}
u\left(-4 u^{2}-2 v^{2}+\alpha\right)=0, \\
v\left(\frac{11}{2} u^{2}+2 v^{2}+\alpha+\beta\right)=0 .
\end{gathered}
$$


It is easily verified that $A D=-8<0, \Delta=3>0$ and $D D_{1}^{\alpha}-A D_{2}^{\alpha}=35 \neq 0$. This means that all the conditions of Theorem 3.1 for a Hopf bifurcation to exist are fulfilled.

The Hopf bifurcation occurs at $\alpha=-\frac{16}{35} \beta$. The invariant circles arising from the Hopf bifurcation are shown in Fig. 1. The four invariant circles come in two pairs with the iterates jumping back and forth between the two invariant circles which have the same sign of $y$.

The equation of the invariant manifold through the trivial fixed point is given by $x=y^{2}+O\left(y^{4}\right)$ as explained in Section 3.3. On this manifold, the dynamics according to (35) is given by

$$
y_{n+1}=y_{n}\left[1+\alpha+\beta+(2+O(\alpha, \beta)) y_{n}^{2}\right]+O\left(y_{n}^{5}\right) \text {. }
$$

As anticipated it can be seen in Fig. 1 that this manifold passes through the two nonsymmetric fixed points.

\section{$4 \quad$ Mode Interactions with $g_{y}^{0}=-1$}

In the previous section, we considered the case where the mode interaction is defined by the conditions $f_{x}^{0}=-1, g_{y}^{0}=1$. A natural question arises as to what happens when $g_{y}^{0}=-1$ which corresponds to a symmetry-breaking period-doubling bifurcation? We now briefly consider this case.

If we define $\tilde{y}_{n}=(-1)^{n} y_{n}$, then it is easy to show that

$$
\begin{aligned}
& x_{n+1}=f\left(x_{n}, \tilde{y}_{n}, \alpha, \beta\right), \\
& \tilde{y}_{n+1}=\tilde{g}\left(x_{n}, \tilde{y}_{n}, \alpha, \beta\right),
\end{aligned}
$$

where $\tilde{g}(x, \tilde{y}, \alpha, \beta)=-g(x, \tilde{y}, \alpha, \beta)$. Thus, if $g_{y}^{0}=-1$, then $\tilde{g}_{\tilde{y}}^{0}=1$ which is the case we have been considering. In both cases the solution structures are the same but the dynamics of the solutions is different.

An alternative approach to this case is to work with equations (5) and to consider bifurcations associated with the irreducible representation $S_{1}=S_{2}=-I$. In this case, 


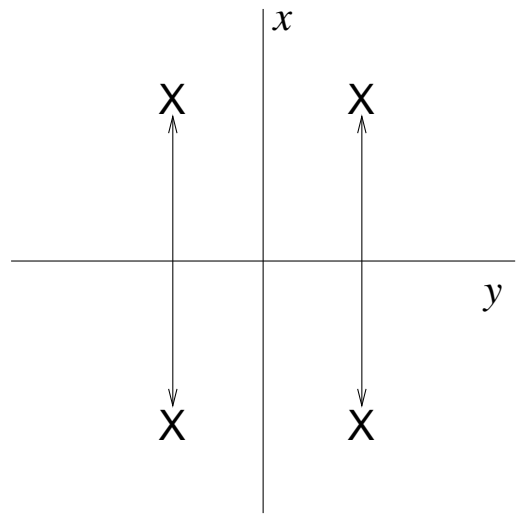

(a)

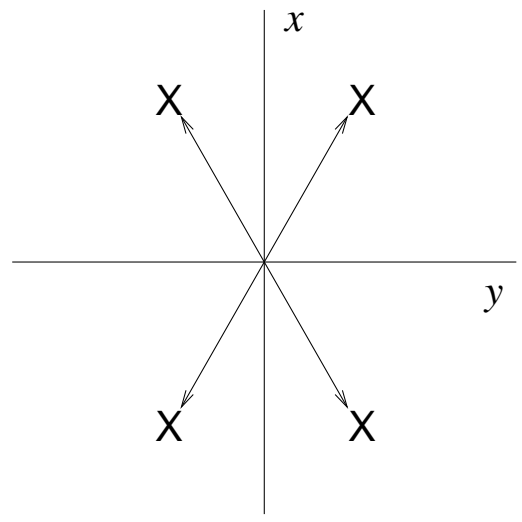

(b)

Figure 2: The dynamics of the two conjugate pairs of non-symmetric period two points when (a) $g_{y}^{0}=1$ and (b) $g_{y}^{0}=-1$.

the bifurcating solutions are fixed by the symmetry $S_{1} S_{2}$ and this corresponds to the symmetry-breaking period-doubling bifurcation associated with $g_{y}^{0}=-1$.

We note that the primary bifurcating solutions in this case have the spatio-temporal symmetry given by $x_{n+1}=x_{n}, y_{n+1}=-y_{n}$. Also, the mixed mode solutions given by (23) have $y_{1}$ and $y_{2}$ with opposite signs in this case. This is illustrated in Fig. 2.

\section{Applications}

The scenario that we have considered of iterated maps with a reflectional symmetry arises in many applications. We consider two such applications here.

\subsection{Two symmetrically coupled maps}

A two-dimensional map with a reflectional symmetry arises naturally from the symmetric coupling of two one-dimensional maps. In this section we apply our previous results to this case.

Suppose that we have two iterations of the form

$$
z_{n+1}=f(z, \lambda), \quad f: \mathbf{R}^{2} \rightarrow \mathbf{R}
$$

which are symmetrically coupled via the function $H(x, y)$. The coupled system is then 
given by

$$
\begin{aligned}
& z_{n+1}^{1}=f\left(z_{n}^{1}, \lambda\right)+c H\left(z_{n}^{1}, z_{n}^{2}\right) \\
& z_{n+1}^{2}=f\left(z_{n}^{2}, \lambda\right)+c H\left(z_{n}^{2}, z_{n}^{1}\right)
\end{aligned}
$$

where $c$ is the coupling parameter. This system has a reflectional symmetry defined by

$$
S\left[\begin{array}{l}
z^{1} \\
z^{2}
\end{array}\right]=\left[\begin{array}{l}
z^{2} \\
z^{1}
\end{array}\right] .
$$

For reasons which we explain later, we assume that the coupling function $H$ satisfies

$$
H(x, y)=-H(y, x)
$$

It follows immediately from this condition that

$$
H(z, z)=0
$$

Differentiating (44), it can be shown that the following relations hold

$$
\begin{gathered}
H_{x}(z, z)=-H_{y}(z, z), \quad H_{x x}(z, z)=-H_{y y}(z, z), \quad H_{x y}(z, z)=0, \\
H_{x x x}(z, z)=-H_{y y y}(z, z), \quad H_{x x y}(z, z)=-H_{x y y}(z, z) .
\end{gathered}
$$

The fixed point space for the symmetry consists of synchronised states where $z_{n}^{1}=z_{n}^{2}$ and the property (45) of the coupling function $H$ ensures that such states are possible for all $n$.

To put the coupled system into the framework that we considered in Section 2, we perform a change of variables defined by

$$
X_{n}=\frac{1}{2}\left(z_{n}^{1}+z_{n}^{2}\right), \quad Y_{n}=\frac{1}{2}\left(z_{n}^{1}-z_{n}^{2}\right) .
$$

The iteration (43) in the new coordinates is given by

$$
\begin{aligned}
X_{n+1} & =\frac{1}{2}\left[f\left(X_{n}+Y_{n}, \lambda\right)+f\left(X_{n}-Y_{n}, \lambda\right)\right] \\
Y_{n+1} & =\frac{1}{2}\left[f\left(X_{n}+Y_{n}, \lambda\right)-f\left(X_{n}-Y_{n}, \lambda\right)+2 c H\left(X_{n}+Y_{n}, X_{n}-Y_{n}\right)\right] .
\end{aligned}
$$


We note that the coupling terms do not appear in the first equation due to the assumption (44). This ensures that the coupling parameter $c$ is a normal parameter, which is what we assumed for the second parameter $\mu$ in Section 2. If we assume that (45) holds but that (44) does not, then the approach that we use still holds but $c$ is no longer a normal parameter.

The symmetry in the new variables is given by

$$
S\left[\begin{array}{l}
X \\
Y
\end{array}\right]=\left[\begin{array}{r}
X \\
-Y
\end{array}\right],
$$

which is in the form that we used in Section 2. The symmetric synchronised subspace now corresponds to the invariant subspace $Y=0$. In this subspace the iteration is given by

$$
X_{n+1}=f\left(X_{n}, \lambda\right)
$$

which is independent of the coupling parameter $c$.

Suppose that we have a path of symmetric fixed points of this system given by $(X, Y)=(\tilde{X}(\lambda), 0)$ on which there is a period-doubling bifurcation at $\lambda=\lambda_{0}$. We perform a change of origin as

$$
X_{n}=\tilde{X}(\lambda)+x_{n}, \quad Y_{n}=y_{n}, \quad \lambda=\lambda_{0}+\alpha
$$

and it is easily verified that

$$
\tilde{X}\left(\lambda_{0}+\alpha\right)=\tilde{X}\left(\lambda_{0}\right)+\frac{f_{\lambda}^{0}}{2} \alpha+O\left(\alpha^{2}\right)
$$

where superscript zero represents evaluation at $\left(\tilde{X}\left(\lambda_{0}\right), \lambda_{0}\right)$. With the above expansion, after ignoring terms of $O\left(\alpha^{2}\right)$ and other higher order terms, and using the relations (46), equations (47) become

$$
\begin{aligned}
x_{n+1}= & -x_{n}+\left(\frac{1}{2} f_{\lambda}^{0} f_{z z}^{0}+f_{z \lambda}^{0}\right) \alpha x_{n}+\frac{1}{2} f_{z z}^{0}\left(x_{n}^{2}+y_{n}^{2}\right)+\frac{1}{6} f_{z z z}^{0}\left(x_{n}^{2}+3 y_{n}^{2}\right) x_{n}, \\
y_{n+1}=y_{n}[ & -1+2 c H_{x}^{0}+\left(f_{z z}^{0}+2 c H_{x x}^{0}\right) x_{n}+\left(\frac{1}{2} f_{\lambda}^{0} f_{z z}^{0}+f_{z \lambda}^{0}+c f_{\lambda}^{0} H_{x x}^{0}\right) \alpha+ \\
& \left.\quad+\left(\frac{1}{2} f_{z z z}^{0}+c\left(H_{x x x}^{0}+H_{x x y}^{0}\right)\right) x_{n}^{2}+\left(\frac{1}{6} f_{z z z}^{0}+c\left(\frac{1}{3} H_{x x x}^{0}-H_{x x y}^{0}\right)\right) y_{n}^{2}\right],
\end{aligned}
$$


where superscript zero denotes evaluation at $\left(\tilde{X}\left(\lambda_{0}\right), \tilde{X}\left(\lambda_{0}\right)\right)$ for the coupling function $H$ and it's derivatives. We note from the second equation that a symmetry-breaking bifurcation is determined by the coefficient $-1+2 c H_{x}^{0}$. Thus, a symmetry-breaking period-doubling bifurcation occurs at the degenerate value $c=0$. However, a symmetrybreaking bifurcation of fixed points occurs when $c=c_{0}=1 / H_{x}^{0}$, assuming that $H_{x}^{0} \neq 0$. Thus, there is a mode interaction at $(\lambda, c)=\left(\lambda_{0}, c_{0}\right)$. We define $c=c_{0}+\beta$ and then equations (49) become

$$
\begin{aligned}
x_{n+1}=-x_{n} & +\left(\frac{1}{2} f_{\lambda}^{0} f_{z z}^{0}+f_{z \lambda}^{0}\right) \alpha x_{n}+\frac{1}{2} f_{z z}^{0}\left(x_{n}^{2}+y_{n}^{2}\right)+\frac{1}{6} f_{z z z}^{0}\left(x_{n}^{2}+3 y_{n}^{2}\right) x_{n}, \\
y_{n+1}=y_{n}[1 & +\left(f_{z z}^{0}+\frac{2 H_{x x}^{0}}{H_{x}^{0}}\right) x_{n}+\left(\frac{1}{2} f_{\lambda}^{0} f_{z z}^{0}+f_{z \lambda}^{0}+\frac{f_{\lambda}^{0} H_{x x}^{0}}{H_{x}^{0}}\right) \alpha+2 H_{x}^{0} \beta \\
& \left.+\left(\frac{1}{2} f_{z z z}^{0}+\frac{H_{x x x}^{0}+H_{x x y}^{0}}{H_{x}^{0}}\right) x_{n}^{2}+\left(\frac{1}{6} f_{z z z}^{0}+\frac{\frac{1}{3} H_{x x x}^{0}-H_{x x y}^{0}}{H_{x}^{0}}\right) y_{n}^{2}\right] .
\end{aligned}
$$

Equations (50) are equivalent to the reduced equations (9) for the coupled system. The coefficients of these equations can be used to find the bifurcation equations (12) with the help of the relations (13). Rather than doing this in general, we consider a specific example.

\section{Example 5.1}

We consider the particular case of linear coupling, with the coupled maps given by

$$
\begin{aligned}
& z_{n+1}^{1}=f\left(z_{n}^{1}, \lambda\right)+c\left(z_{n}^{2}-z_{n}^{1}\right) \\
& z_{n+1}^{2}=f\left(z_{n}^{2}, \lambda\right)+c\left(z_{n}^{1}-z_{n}^{2}\right)
\end{aligned}
$$

Clearly $H(x, y)=y-x$ in this case and $H_{x}^{0}=-H_{y}^{0}=-1$, with all the other derivatives of $H$ being zero. Thus, the mode interaction occurs when $c_{0}=-1$. The reduced equations (50) then become

$$
\begin{aligned}
& x_{n+1}=-x_{n}+\left(\frac{1}{2} f_{\lambda}^{0} f_{z z}^{0}+f_{z \lambda}^{0}\right) \alpha x_{n}+\frac{1}{2} f_{z z}^{0}\left(x_{n}^{2}+y_{n}^{2}\right)+\frac{1}{6} f_{z z z}^{0}\left(x_{n}^{2}+3 y_{n}^{2}\right) x_{n}, \\
& y_{n+1}=y_{n}\left[1+f_{z z}^{0} x_{n}+\left(\frac{1}{2} f_{\lambda}^{0} f_{z z}^{0}+f_{z \lambda}^{0}\right) \alpha-2 \beta+\frac{1}{6} f_{z z z}^{0}\left(3 x_{n}^{2}+y_{n}^{2}\right)\right] .
\end{aligned}
$$

Comparing equations (52) with equations (9) gives the coefficients of the reduced equations. Using (13) we can calculate the coefficients of the bifurcation equations (12) which 
are given by

$$
\begin{array}{ccc}
A=-\frac{1}{12}\left[3\left(f_{z z}^{0}\right)^{2}+2 f_{z z z}^{0}\right], & B=\frac{1}{4}\left[\left(f_{z z}^{0}\right)^{2}-2 f_{z z z}^{0}\right], \\
C=-\frac{1}{4}\left[\left(f_{z z}^{0}\right)^{2}-2 f_{z z z}^{0}\right], & D=\frac{1}{12}\left[3\left(f_{z z}^{0}\right)^{2}+2 f_{z z z}^{0}\right], \\
a_{2}=-\left(\frac{1}{2} f_{\lambda}^{0} f_{z z}^{0}+f_{z \lambda}^{0}\right), \quad a_{3}=0, & b_{2}=\frac{1}{2} f_{\lambda}^{0} f_{z z}^{0}+f_{z \lambda}^{0}, \quad b_{3}=-2 .
\end{array}
$$

The nondegeneracy conditions defined by (16) and (17) are given by

$$
\begin{aligned}
A & =-\frac{1}{12}\left[3\left(f_{z z}^{0}\right)^{2}+2 f_{z z z}^{0}\right] \neq 0, \\
D & =\frac{1}{12}\left[3\left(f_{z z}^{0}\right)^{2}+2 f_{z z z}^{0}\right] \neq 0 \\
a_{2} & =-\left(\frac{1}{2} f_{\lambda}^{0} f_{z z}^{0}+f_{z \lambda}^{0}\right) \neq 0 \\
b_{2} & =\frac{1}{2} f_{\lambda}^{0} f_{z z}^{0}+f_{z \lambda}^{0} \neq 0 . \\
\Delta & =-\frac{1}{9}\left[3\left(f_{z z}^{0}\right)^{2}-2 f_{z z z}^{0}\right] f_{z z z}^{0} \neq 0, \\
b_{2} A-a_{2} C & =-\frac{2}{3}\left[\frac{1}{2} f_{\lambda}^{0} f_{z z}^{0}+f_{z \lambda}^{0}\right] f_{z z z}^{0} \neq 0 \\
b_{2} B-a_{2} D & =\frac{1}{6}\left[\frac{1}{2} f_{\lambda}^{0} f_{z z}^{0}+f_{z \lambda}^{0}\right]\left[3\left(f_{z z}^{0}\right)^{2}-2 f_{z z z}^{0}\right] \neq 0 \\
b_{2} a_{3}-a_{2} b_{3} & =2\left(\frac{1}{2} f_{\lambda}^{0} f_{z z}^{0}+f_{z \lambda}^{0}\right) \neq 0 .
\end{aligned}
$$

These conditions are all satisfied if

$$
3\left(f_{z z}^{0}\right)^{2}+2 f_{z z z}^{0} \neq 0, \quad \frac{1}{2} f_{\lambda}^{0} f_{z z}^{0}+f_{z \lambda}^{0} \neq 0, \quad 3\left(f_{z z}^{0}\right)^{2}-2 f_{z z z}^{0} \neq 0, \quad f_{z z z}^{0} \neq 0 .
$$

Note that the condition $f_{z z z}^{0} \neq 0$ implies that a quadratic map $f$ (such as the logistic map) does not fulfil all of these conditions. If all of these nondegeneracy conditions are satisfied, then there is a non-degenerate mode interaction and the results of the previous sections can be applied to the coupled system (51).

Other couplings such as quadratic coupling $\left(H(x, y)=y^{2}-x^{2}\right)$ or coupling of outputs $(H(x, y, \lambda)=f(y, \lambda)-f(x, \lambda))[19]$ could be considered in a similar way. Note that in the latter case, the coupling function also depends on the parameter $\lambda$, and so a few additional terms would occur in the reduced equations (50) in this case. 


\subsection{Two coupled vertically forced pendulums}

A practical example of a coupled system which exhibits the type of mode interaction we have studied is a system of two coupled damped pendulums subject to vertical forcing. Period-doubling bifurcations for the single pendulum (which corresponds to motion in the reflection-invariant synchronised subspace) together with symmetry-breaking bifurcations for the coupled system have been found by Kim and Lee [20].

The equations of motion for the coupled system are given by [20]

$$
\begin{aligned}
& \ddot{x}_{1}=f\left(x_{1}, \dot{x}_{1}, t\right)+c g\left(x_{1}, x_{2}\right), \\
& \ddot{x}_{2}=f\left(x_{2}, \dot{x}_{2}, t\right)+c g\left(x_{2}, x_{1}\right),
\end{aligned}
$$

where

$$
f(x, \dot{x}, t)=-2 \pi\left[\gamma \dot{x}+\left(\omega_{0}^{2}-A \cos 2 \pi t\right) \sin 2 \pi x\right], \quad g\left(x_{1}, x_{2}\right)=\frac{1}{2}\left(x_{2}-x_{1}\right) .
$$

The quantities $2 \pi x_{1}$ and $2 \pi x_{2}$ are the angles of the two pendulums from the downward vertical and the parameters are $\gamma$, the damping coefficient, $\omega_{0}$, the natural frequency of the pendulum, $A$, the amplitude of the forcing and $c$, the coupling parameter. Following [20], we choose $\gamma=0.1$ and $\omega_{0}=0.5$ and vary the parameters $A$ and $c$.

Synchronised motion of this coupled system is given by $x_{1}=x_{2}=x$, and corresponds to motion of the single pendulum. This has the trivial solution $x(t)=0$ that is stable for small values of $A$. As $A$ increases, this solution loses stability at $A=0.10022$ to a periodic orbit of period 2 (twice the period of the forcing) with the symmetry property $x(t+1)=-x(t)$. This symmetry is broken at a bifurcation which occurs at $A=0.33526$, resulting in a periodic orbit of period 2 with no additional properties. It is on this branch that we find a mode interaction.

This solution undergoes a period-doubling bifurcation at $A=0.35416$. Rewriting the two second order differential equations as four first order equations and sampling the trajectory at $t_{n}=2 n, n=0,1,2, \ldots$ i.e. once for every two cycles of the forcing, gives a four-dimensional map in $Z_{n}=\left(x_{1}\left(t_{n}\right), \dot{x}_{1}\left(t_{n}\right), x_{2}\left(t_{n}\right), \dot{x}_{2}\left(t_{n}\right)\right)$. Thus, the period-doubling 

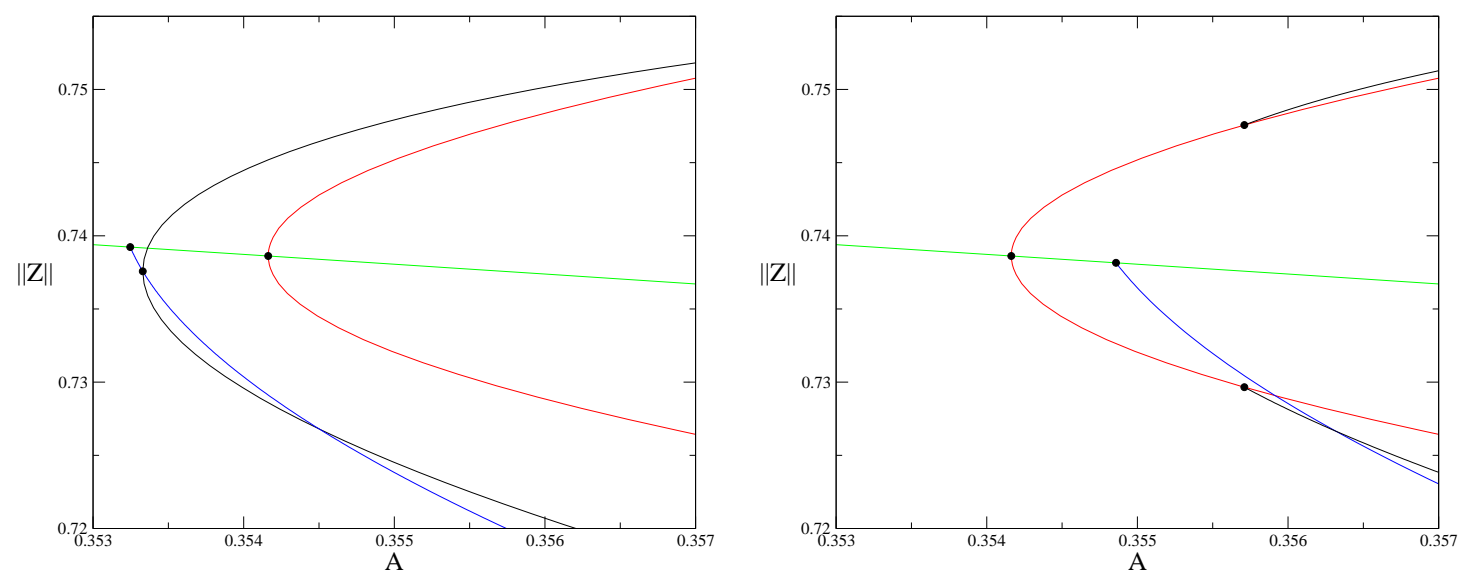

Figure 3: Solutions for the coupled pendulum problem at $c=4.26$ (before the mode interaction) and at $c=4.35$ (after the mode interaction). The branches are symmetric period 1 (green), symmetric period 2 (red), non-symmetric period 2 with spatio-temporal symmetry (blue), non-symmetric period 2 (black).

bifurcation at $A=0.35416$ involves a period 1 orbit of this map bifurcating to a period 2 orbit.

For the coupled system, the period-doubling bifurcation does not depend on the coupling parameter $c$ as it is a normal parameter. There are in fact three mode interactions involving the period-doubling bifurcation and a symmetry-breaking bifurcation, and these occur at $c_{1}=4.3119, c_{2}=0$ and $c_{3}=-1.0510$. The first two of these involve a symmetry-breaking period-doubling bifurcation (as discussed in Section 4), while the third involves a symmetry-breaking bifurcation of fixed points. Solutions before and after the first mode interaction at $c_{1}=4.3119$ were computed using AUTO [21] and are shown in Fig. 3. We note that the geometry of the solution branches together with knowledge of the stability of the symmetric period 1 branch can be used to show that there is no tertiary Hopf bifurcation for this mode interaction [16].

\section{Conclusions}

We have studied mode interactions in systems with a reflectional symmetry which involve a symmetry-preserving period-doubling bifurcation and a symmetry-breaking bifurcation, which may give rise to a branch of either non-symmetric fixed points or nonsymmetric period two points. A centre manifold reduction for such a mode interaction 
has an additional $Z_{2}$ normal form symmetry, but this exists only up to finite order in the normal form and the effect of tail terms (which may break the symmetry) must be considered. To avoid these problems, we have constructed an enlarged system which has both fixed points and period 2 points as solutions, and which has an additional reflectional symmetry, and have used Lyapunov-Schmidt reduction to obtain $Z_{2} \times Z_{2}$ symmetric bifurcation equations at the mode interaction. Since these bifurcation equations are only algebraic, they cannot provide any dynamic information beyond the existence of period 2 orbits. We therefore derived various dynamic properties of the problem, including the existence of an invariant manifold between the trivial fixed point and the non-symmetric fixed points, and conditions for the existence of a tertiary Hopf bifurcation on the mixed mode solutions.

These techniques were applied to two symmetrically coupled maps, and also to the dynamics of two coupled, vertically forced pendulums, which are described by a fourdimensional Poincaré map.

Finally, we note that if the symmetric period-doubling bifurcation is the start of a period-doubling cascade, then in the two parameter setting, the secondary symmetrybreaking bifurcation which arises from the mode interaction may continue up the branch of symmetric period 2 points, and then be involved in another mode interaction with the second period-doubling bifurcation. In this way, an infinite cascade of mode interactions can occur. We study this problem in a subsequent paper [22].

\section{Acknowledgements}

The authors are grateful to the referees for their comments which have improved this paper.

\section{References}

[1] W.F. Langford and G. Iooss. Interactions of Hopf and pitchfork bifurcations. Bifurcation Problems and Their Numerical Solution, (Proc. Workshop, Univ. Dortmund, 
Dortmund, 1980), Internat. Ser. Numer. Math. 54, Birkhäuser, Basel-Boston, Mass., 103-134, 1980.

[2] M. Golubitsky and D.G. Schaeffer. Singularities and Groups in Bifurcation Theory. Vol. I, Appl. Math. Sci. 51, Springer-Verlag, New York, 1985.

[3] D. Armbruster and G. Dangelmayr. Coupled stationary bifurcations in nonflux boundary value problems. Math. Proc. Camb. Phil. Soc. 101, 167-192, 1987.

[4] M. Golubitsky, I. Stewart and D.G. Schaeffer. Singularities and Groups in Bifurcation Theory. Vol. II, Appl. Math. Sci. 69, Springer-Verlag, New York, 1988.

[5] S.B.S.D. Castro. Mode interactions with spherical symmetry. Int. J. Bif. Chaos 4, 885-904, 1994.

[6] Yu.A. Kuznetsov, H.G.E. Meijer and L. van Veen. The fold-flip bifurcation. Int. J. Bif. Chaos 14, 2253-2282, 2004.

[7] Yu.A. Kuznetsov and H.G.E. Meijer. Numerical normal forms for codim 2 bifurcations of fixed points with at most two critical eigenvalues. SIAM J. Sci. Comp. 26, 1932-1954, 2005.

[8] Yu.A. Kuznetsov and H.G.E. Meijer. Remarks on interacting Neimark-Sacker bifurcations. J. Diff. Eqns Appls 12, 1009-1035, 2006.

[9] P. Chossat and M. Golubitsky. Iterates of maps with symmetry. SIAM J. Math. Anal. 19, 1259-1270, 1988.

[10] P.J. Aston. An investigation of a mode interaction involving period-doubling and symmetry-breaking bifurcations. Dynamics, Bifurcation and Symmetry, New Trends and New Tools, ed. P. Chossat, Kluwer, 49-58, 1994.

[11] I. Melbourne. Derivation of the time-dependent Ginzburg-Landau equation on the line. J. Nonlin. Sci. 8, 1-15, 1998. 
[12] B. Peckham and I.G. Kevrekidis. Period doubling with higher order degeneracies. SIAM J. Math. Anal. 22, 1552-1574, 1991.

[13] P. Ashwin, J. Buescu and I. Stewart. From attractor to chaotic saddle: a tale of transverse instability. Nonlinearity 9, 703-737, 1996.

[14] D. Ruelle. Bifurcations in the presence of a symmetry group. Arch. Rat. Mech. Anal. 51, 136-152, 1973.

[15] G. Cicogna and G. Gaeta. Symmetry invariance and centre manifolds for dynamical systems. Nuovo Cimento B 109, 59-76, 1994.

[16] F. Amdjadi and P.J. Aston. Detection of tertiary Hopf bifurcations arising from mode interactions. Int. J. Bif. Chaos 7, 1691-1698, 1997.

[17] S. Wiggins. Introduction to Applied Nonlinear Dynamical Systems and Chaos, Texts in Applied Mathematics 2, Springer-Verlag, New York, 1990.

[18] D.K. Arrowsmith and C.M. Place. An Introduction to Dynamical Systems, CUP, Cambridge, 1990.

[19] H. Mir. Cascading Mode Interactions in Discrete Dynamical Systems, PhD thesis, University of Surrey, 2007.

[20] S.Y. Kim and K. Lee. Period doublings in coupled parametrically forced damped pendulums. Phys. Rev. E 54, 1237-1252, 1996.

[21] E.J. Doedel, R.C. Paffenroth, A.R. Champneys, T.F. Fairgrieve, Yu.A. Kuznetsov, B. Sandstede and X. Wang. AUTO 2000: Continuation and bifurcation software for ordinary differential equations (with HomCont). Technical Report, Caltech, 2001.

[22] H. Mir and P.J. Aston. Mode interaction cascades in iterated maps. In preparation. 


\section{Figure Captions}

Fig. 1. Phase portrait for $\alpha=0.0683$ and $\beta=-0.1 . \quad \times-$ fixed points, o - period 2 points.

Fig. 2. The dynamics of the two conjugate pairs of non-symmetric period two points when (a) $g_{y}^{0}=1$ and (b) $g_{y}^{0}=-1$.

Fig. 3. Solutions for the coupled pendulum problem at $c=4.26$ (before the mode interaction) and at $c=4.35$ (after the mode interaction). The branches are symmetric period 1 (green), symmetric period 2 (red), non-symmetric period 2 with spatiotemporal symmetry (blue), non-symmetric period 2 (black). 